\title{
Tracheotomy, closure of long-term tracheostomy and standard tracheal segmental resections
}

\author{
Alper Toker, J. Awori Hayanga, Ankit Dhamija, Robert Herron, Ghulam Abbas \\ Department of Cardiovascular and Thoracic Surgery, West Virginia University, Heart and Vascular Surgery, Morgantown, WV, USA \\ Contributions: (I) Conception and design: A Toker; (II) Administrative support: A Dhamija, R Herron; (III) Provision of study materials or patients: \\ A Toker; (IV) Collection and assembly of data: A Toker; (V) Data analysis and interpretation: JA Hayanga; (VI) Manuscript writing: All authors; \\ (VII) Final approval of manuscript: All authors. \\ Correspondence to: Alper Toker. 1 Medical Center Drive Morgantown, WV 26506, USA. Email: salpertoker@gmail.com; alper.toker@wvumedicine.org.
}

\begin{abstract}
Tracheotomy is a surgical procedure commonly employed to establish stable and long-term airway access. Iatrogenic airway injury post procedure may have serious consequences with limited treatment options. Tracheostoma or long standing tracheostomies require special closing techniques. Tracheotomies, tracheostomies, complications of these and treatment options, long standing tracheostomy closure techniques, and standard tracheal segmental resections are discussed.
\end{abstract}

Keywords: Tracheostomy; closure of tracheostomy; tracheal resections

Submitted Dec 13, 2019. Accepted for publication Jan 30, 2020.

doi: $10.21037 /$ jtd.2020.02.41

View this article at: http://dx.doi.org/10.21037/jtd.2020.02.41

\section{Tracheotomy}

Tracheotomies have been performed for thousands of years, the first of which was carried out in Egypt in 3600 BC (1). Aretaeus (2nd century AD) and Galen (2nd to 3rd century $\mathrm{AD})$ wrote that tracheostomy was performed by Asklepiades around $100 \mathrm{BC}$ (2). Dr. Jackson standardized the currently used technique in 1909 (3). Open technique has essentially almost remained the same, with development of new percutaneous techniques, cannulae and equipment within the last several decades.

\section{Terminology}

'Tracheotomy' refers to a surgical procedure in which an inicison is made into the trachea to allow direct access to this tube, typically to maintain a patent airway, provide ventilation, and remove secretions by suctioning as needed. Tracheostomy is the resulting hole or 'stoma' created by such an incision. Tracheostomies may be temporary or permanent; short- or long-term. The closure of longterm tracheostomies deserves particular attention, as if not closed properly, a tracheocutaneous fistula may develop, which prevents closure of the artificially created airway, leading to complications such as pneumothorax and pneumomediastinum, loss of protective skin integrity, and body image issues. Tracheal resection may be required for both benign and malignant tracheal conditions.

The aim of this paper is to discuss surgical tracheotomy performance, as well as closure of long-term tracheostomy and standard segmental tracheal resection.

\section{Anatomic overview of the trachea}

The trachea is one of the organs of the body which rarely shows anatomic variation. However, its shape and anatomic location may vary with changes in body habitus. Cervical flexion and extension also affects positioning; when the neck is extended, the trachea is pulled up out of the thoracic cavity and when the neck is flexed, it moves to an intrathoracic position.

The trachea is a semiflexible tube 1.5 to $2 \mathrm{~cm}$ in width and 10 to $13 \mathrm{~cm}$ in length. Anatomically, it begins at the 6th to 7 th cervical vertebra and divides at the 4th to 5 th thoracic vertebra levels, extending from the lower larynx to the carina. It generally has 20 cartilaginous inverse U-shaped 
rings, incomplete posteriorly, embedded in a fibrous membrane of elastic tissue. The trachealis muscle connects these cartilaginous rings and contains both transverse and longitudinal fibers. Tracheal glands are located in the connective tissue between epithelium and cartilage. These glands lubricate the inner surface of the trachea, which consists of pseudostratified columnar epithelium. This epithelium contains kinocilia, the motion of which provides transport of mucous and other particles to the larynx for excretion by coughing.

The brachiocephalic artery crosses the anterior surface of the trachea behind the sternal manubrium, but when the neck is hyperextended, it may be located close to the sternal notch. In women who are short in stature or overweight, the brachiocephalic artery may be completely contained within the neck. The esophagus and cervical spine are posterior to the trachea. The bilateral recurrent laryngeal nerves run along the lateral aspects of the trachea longitudinally. The parathyroid glands and carotid arteries run around the trachea. The thyroid gland is located around and unites at the anterior trachea with an isthmus at the cervical level.

The subglottic space extends from the inferior margin of the vocal cords to the lower border of cricoid cartilage; this area is the narrowest part of the airway. The subglottic space is vulnerable to mechanical trauma, particularly intubation of longer duration. The laryngeal nerves enter the larynx through the posterior cricoid cartilage ring. The resection of the entire cricoid cartilage ring is impossible, using the current techniques available, without damaging both recurrent laryngeal nerves. Such damage would result in total loss of bilateral vocal cord function. These characteristics of the trachea pose a challenge to thoracic surgeons performing tracheotomies, tracheal closures, or resections.

\section{Tracheostomy}

A tracheostomy is a temporary or permanent tracheal window created in the lower anterior neck, above the sternal notch. It bypasses the upper airway. Indications for tracheostomy include the need to secure the airway provide ventilation, and clear secretions in the event orotracheal intubation cannot be performed, or when invasive mechanical ventilation will be required for a long duration, typically more than 1-2 weeks. It also more enhances patient comfort when compared to orotracheal intubation, prevents skin breakdown of the face, and allows for more facile patient communication and better oral hygiene.
In contemporary airway medicine, tracheostomy should be an elective surgery. If endotracheal intubation is initially precluded by stenosis or presence of a mass, dilating the trachea with ventilating rigid bronchoscopy or coring out a portion of tumor may then allow for performance of endotracheal intubation. In acute settings, tracheotomy may be reserved for relief of mechanical airway obstruction which could not be treated with rigid bronchoscopy and endotracheal treatment modalities.

In subacute settings, tracheotomy may be performed to resect laryngeal cancer or a long segment tracheal stenosis, if the location and characteristics of the lesion are not suitable for a surgical reconstruction. In the case of long segment tracheal stenosis, tracheotomy should be done only by experienced (thoracic or ENT) surgeons, so as not to lose a subsequent opportunity for reconstruction. There are almost no absolute contraindications to performance of tracheotomy, however, some conditions require an experienced surgical team and include substernal goiter, severe kyphosis, anatomic defects, soft tissue infections, and bleeding diathesis.

\section{Concerns about a tracheostomy}

Open and percutaneous tracheostomy procedures carry different risks. Open tracheostomy, although considered a safe procedure, has potential side complications, categorized as occurring in immediate, early, or late from the time of the index surgery. Immediate complications include bleeding and membranous tracheal injury, and tracheal stenosis or malacia are late complications (4). Other complications related to a tracheostomy include pneumothorax, subglottic stenosis, tracheoesophageal fistula (TEF), vocal cord dysfunction, stomal granulation, persistent tracheacutanous fistula, and scarring. The indications, technique, timing, and selection criteria are thought to be reasons in for complications in critically ill patients (5). Tracheostomy complications can be considered in 3-time frames: immediate, early, and late (Table 1) $(4,5)$.

\section{Optimum timing of tracheostomy in respiratory failure}

Optimal timing of tracheostomy based on recent randomized controlled trials are controversial. Results of two recent multi-center, randomized trials $(6,7)$ found that clinicians could not accurately predict which patients would require prolonged mechanical ventilation, and that an early 
Table 1 Complications of tracheostomy

\begin{tabular}{lll}
\hline Immediate complications & Early complications & Late complications \\
\hline Apnea & Bleeding & Bleeding \\
Injury to nearby tissues (great vessels, esophagus and laryngeal nerves & Displacement/dislodging & Tracheal stenosis \\
Bleeding & Mucous plugging & Trachea cutanous fistula \\
Tracheostomy placement in mediastinum & Subcutaneous emphysema & Tracheoesophageal fistula \\
Pneumothorax & Tracheitis & Trachea innominate artery fistulas \\
Postobstructive pulmonary edema & Wound infection & Tracheomalacia \\
Tearing of trachea & Myiasis & Wound infection \\
\hline
\end{tabular}

tracheotomy did not have more benefits than the standard planning. Based on the data obtained until today the optimal time to consider tracheotomy is 10 days, although every effort should be made to ensure necessity of such a surgery, such as conduction of spontaneous breathing trials, assessment of the patient's ability to protect the airway, and secretion management $(7,8)$.

\section{Open surgical versus percutaneous tracheostomy}

It has been generally accepted that in adult ICU patients who do not have a contraindication to percutaneous tracheostomy, a percutaneous dilatational tracheostomy (PDT) would be considered the procedure of choice. In a systematic review and meta-analysis of 17 research clinical trials (RCT), it was reported that wound infection is reduced in PDT. No differences in bleeding, major periprocedural complications and long-term complications between PDT and open tracheostomy procedures were reported (9).

In another meta-analysis which includes 15 RCTs and nearly 1,000 subjects, comparison of open tracheotomy versus PDT demonstrated significantly fewer complications in the PDT group in terms of wound infection and unfavorable scarring. Authors were unable to show significant differences for complications including false passage, minor bleeding or major hemorrhage, subglottic stenosis, and mortality. It has been shown significantly that, a PDT was associated with a higher rate of decannulation/ obstruction which has higher potential for mortality. PDT cases takes shorter in duration and associated with reduced cost (\$456 less) when compared with open surgical tracheostomy (10). However, despite these benefits, some surgeons still prefer the technique of open tracheostomy.

\section{High-risk patients and PDT}

Complication rates for PDT in high-risk patients with coagulopathy, thrombocytopenia, and other bleeding diatheses, with or without correction at the discretion of the physician performing the tracheostomy have been rarely reported (11-13). Correction of coagulopathy is recommended in the periprocedural period to allow for safe PDT in the coagulopathic patients. Obesity is another risk factor which has been reported to be an indication to opt for an open surgical tracheotomy over PDT. A number of small retrospective studies found no difference in complication rates with the PDT procedure in obese patients (14-18).

In patients with risk factors, the surgeon must consider the neck anatomy. In a patient with obesity or coagulopathy the decision to do percutaneous tracheostomy should take these factors into account such as; the person patient's neck anatomy and experience of the performing physician. The practitioner must be prepared to convert to the open surgical technique if complications are encountered during the PDT procedure.

\section{Preoperative evaluation and points to consider}

The trachea generally meets the sternum at a 45-degree angle in the thoracic inlet. In patients with kyphosis this angle may increase up to 90 degrees (Figure 1). This angle may be underestimated and may cause misplacement of the cannulae into the mediastinum. In the usual surgical practice, the 2 nd and $3 \mathrm{rd}$ tracheal rings are selected as the tracheostomy site. In the elderly, kyphotic, and obese 


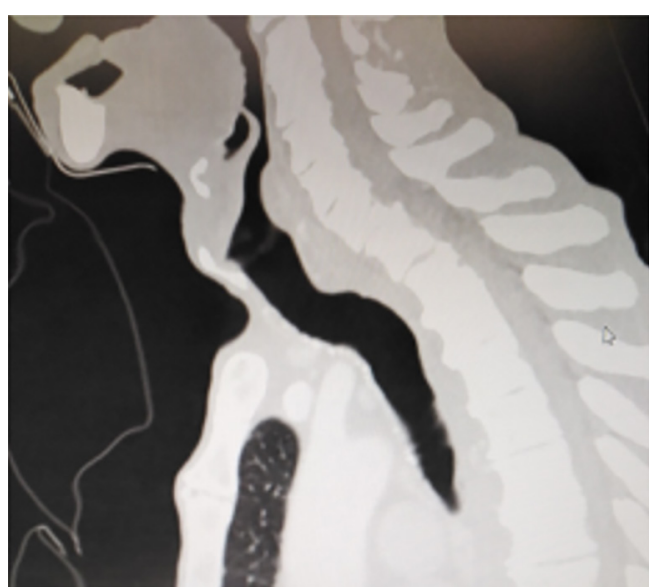

Figure 1 Trachea of an elderly kyphotic patient. The angle between sternum and trachea is almost 90 degrees.

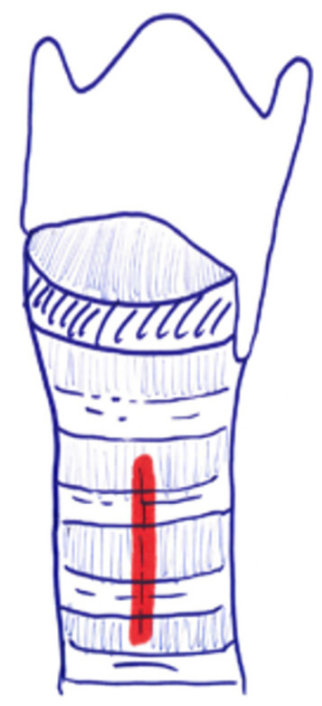

Figure 2 Vertical tracheotomy over the 2 nd and 3 rd cartilages.

patients these rings may be located behind the sternum. As the neck is extended, the trachea rises away from the sternum. This allows a greater space in which to incise for tracheotomy, however, this extension may increase the likelihood of encountering the thyroid isthmus.

From a vascular perspective, two vessels are key, the thyroid ima and the brachiocephalic trunk. Thyroid ima ascends in the neck to supply the blood for the inferior thyroid pole and gland. This artery may have anatomic differences in origin. It is usually separated from the brachiocephalic artery, but in $10 \%$ of the population, it may also originate directly from the aorta, with risk for bleeding. The brachiocephalic artery may be raised up to sternal notch in some patients. Pre-operative correction of coagulopathy should be considered, with a goal of a platelet count of at least 50,000 thrombocytes/mL and the patient should not be receiving antiplatelet pharmacotherapy, with such medications as clopidogrel, warfarin, or apixaban should have an INR less than 1.6 at the time of surgery. If the patient is ventilated with an $\mathrm{FiO}_{2}$ greater than 0.60, extra care for hemostasis should be given during the tracheotomy because repair of a bleeding vessel may require seperation from ventilation. A recent sternotomy (in the past 10 days) has been always in the middle of the debate for tracheostomy opening. However, literature findings do not support this claim (19). Special attention may be needed for patients who are supported by extracorporeal membrane oxygenation (ECMO). This is not different any other patient who receives anticoagulation medication. Patients who have burns require special consideration. It is generally recommended that tracheotomy may be performed either through an autograft or more than 10 days after the skin grafting procedure to decrease infectious complications (20).

\section{Operative technic for tracheostomy}

Grillo describes tracheotomy in his technical summary (21). He suggests planning site of the incision based on cricoid cartilage. Thyroid isthmus is generally found on the second cartilage ring. Division and suture ligation of the thyroid isthmus are recommended. This maneuver provides full exposure from cricoid membrane to the 4th cartilage. Next, a vertical incision through the 2 nd to $3 \mathrm{rd}$ rings should be made anteriorly (Figure 2). Inverse U-shaped flap tracheostomy (Figure 3) or removal of a portion of tracheal ring to create a trapdoor should be avoided, as these maneuvers cause tracheal stenosis, tracheomalacia and further complications related to tracheostomy. In the current literature, U-shaped incisions (Bjork Flap), H shaped incisions or removal of a circular fragment of the anterior wall of the trachea are reported to have a high incidence of tracheal stenosis and for this reason they are generally no longer used. Regardless of the type of tracheal incision, the principle rule is leaving the first cartilage ring intact and not cutting down to the 3 rd cartilage ring due to risk of brachiocephalic artery fistula development (21).

\section{Complications of tracheostomy}

The two most common complications of both open and 
percutaneous techniques are bleeding and dislodgment of the tracheostomy cannula. Inadvertent dislodgment of a cannula can be fatal due to obstruction in the early postoperative period if not corrected. If the dislodged cannula cannot be reinserted, then urgent orotracheal intubation is required, with replacement of the cannulae under safer conditions.

Infection is another risk. It may be unavoidable due to bacterial colonization of the trachea. If the patient develops cellulitis or a wound infection around the tracheostomy site, a devasting necrotizing pneumonia may develop. If this occurs, the patient should be kept intubated and debridement of the tracheostomy site should be performed, along with the initiation of targeted pharmacologic therapy and meticulous wound care.

Tracheostomy complications are adopted and modified

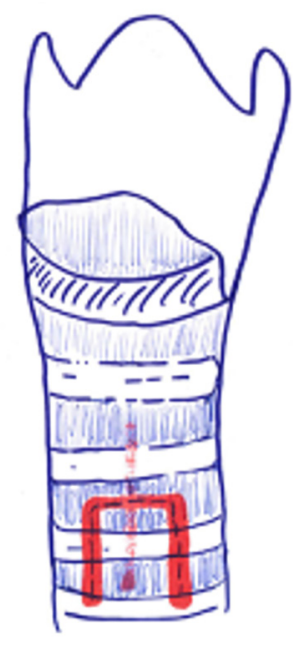

Figure 3 Flap tracheotomies are not recommended due to possible long-term complications. from Shields TW, 2019 edition (22). Follow-up parameters are demonstrated in Table 2.

In analysis of data from a single institution (23) it was demonstrated that the complication rate is low when tracheotomies are performed by experienced surgeons. The most common long-term complication was reported to be tracheal stenosis. Authors claim that, stenosis diagnosed in their patients may be due to prolonged intubation prior to the opening of a tracheotomy. Out of 1,130 patients, they reported that tracheal stenosis occurred in $1.8 \%$, hemorrhage in $0.8 \%$, tracheocutaneous fistula in $0.5 \%$, infection in $0.4 \%$, inadvertent decannulation/obstruction in $0.3 \%$ and tracheo-esophageal fistula (TEF) in $0.08 \%$ (23).

Some late complications of tracheostomy is due to necrosis of the surrounding structures, which may occur days to years post procedure. There are 3 important types of tracheal fistulas: tracheoinnominate artery fistula (TIF), TEF, and persistent tracheocutaneous fistula (PTCF).

TIF a may occur at longer than 1 week post tracheotomy. If bright red blood is visualized in or around the stoma, the site should be immediately inspected. If TIF is suspected, then the patient should be further evaluated in the operating room, with immediate ability to repair surgically (Figures 4,5 ). If TIF is unlikely, then the examination may be performed with fiber-optic bronchoscopy, after temporary decannulation.

TEF is generally associated with another foreign body positioned inside the esophagus such as a nasogastric tube or stent. Assessment of findings associated with TEF include bile tinged secretions, massive air leak during mechanical ventilation, inflation of nasogastric drainage bag in mechanically ventilated patients and recurrent or persistent pneumonia. The diagnosis may be confirmed by modified barium swallow study, oral contrast-enhanced chest computerized axial tomography (CT), esophagoscopy,

Table 2 Follow-up for different periods of tracheostomy

\begin{tabular}{lll}
\hline Early follow-up & Intermediate follow-up & Late follow-up \\
\hline $\begin{array}{l}\text { End-tidal } \mathrm{CO}_{2} \\
\text { Chest X-ray }\end{array}$ & $\begin{array}{l}\text { Physical examinations during } \\
\text { cannula replacement, Fiberoptic } \\
\text { tracheoscopies }\end{array}$ & Take serious every symptom bleeding and aspiration \\
Bronchoscopy uses during PDT & & Contrast enhanced CT \\
Chest CT contrast enhanced & Barium swallow \\
& Highly suspicious conditions angiography \\
\hline
\end{tabular}




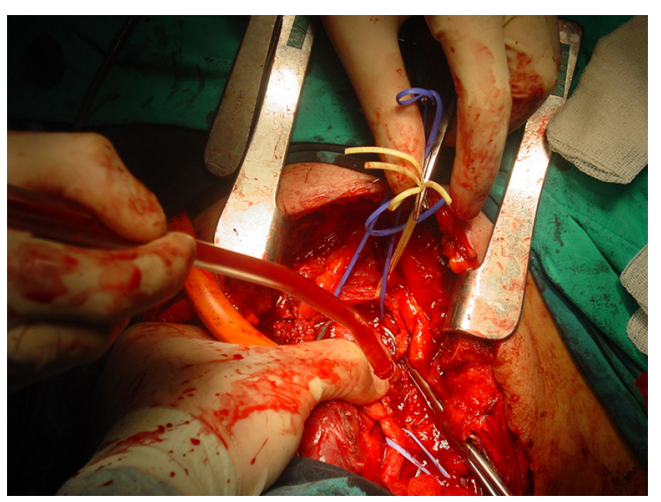

Figure 4 Surgeon performs most of the procedure with one hand compressing the innominate artery and making the required dissections with the other hand until everything is under control.

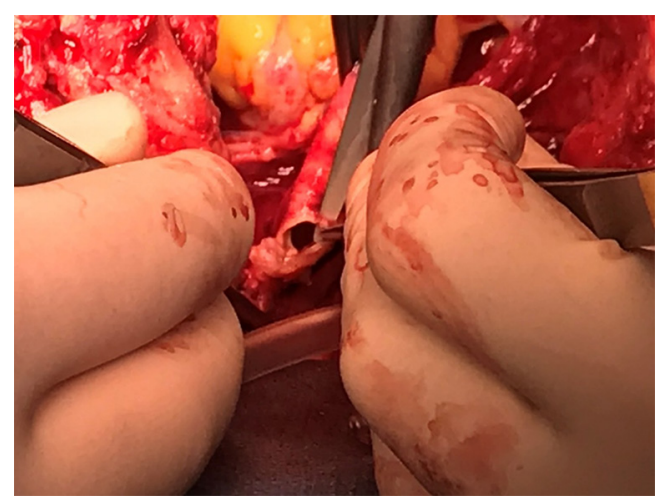

Figure 5 Identification of the defect, closure or division but definitely exclusion from trachea is recommended. Some authors recommend to leave the fistoulous artery on the trachea by dividing the vessel from proximal and distal to the defect.
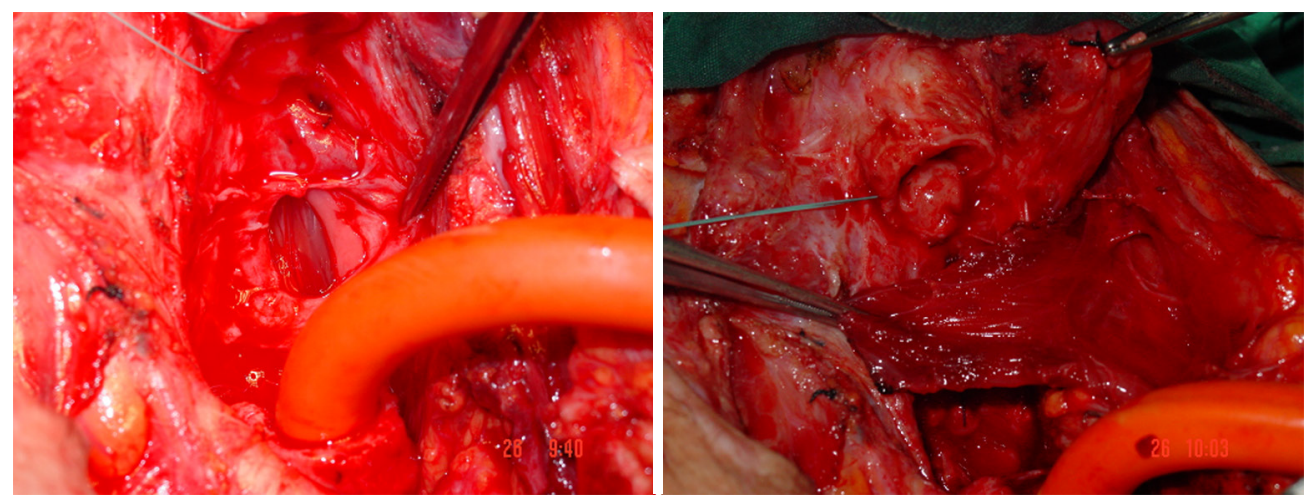

Figure 6 For the treatment of tracheoesophageal fistula resection of destructed trachea, primary closure of esophagus and muscle interposition are the mainstem of the treatment.

or bronchoscopy. For the treatment of TEF resection of destructed trachea, primary closure of esophagus and muscle interposition are the mainstem of the treatment (Figure 6).

A tracheocutanous fistula is a pathologic complication that occurs post decannulation, in which the tracheostomy tract does not close spontaneously. If the stoma is still open within 6 months after decannulation, despite closure efforts, due to epithelization of the tracheostomy tract, this is called tracheocutanous fistula. These are two different definitions. In the first one, tracheostomy does not close spontaneously. In the second, it remains open "despite closure efforts". This situation requires resection of the skin growth along the stoma. This condition may need primary closure. Closure of PTCF techniques include fistulectomy by multilayered closing of the deepithelized local tissue and/or major reconstructive surgeries (24-32). Complete closure of the tracheal defect may not be possible by simple resection and primary closure of layers. When positive pressures are generated by coughing or speaking, the sutures may dehisce and air may penetrate the layers and become trapped in the surrounding tissues in the facial planes of the neck and chest, as evidenced by edema and subcutaneous emphysema. Infection may follow the spread of contaminated air, blood, and secretions $(33,34)$.

Pneumothorax, pneumomediastinum, and respiratory compromise may ensue. Other potential complications of surgical closure techniques include local wound infection, bleeding, hematoma or seroma formation, and suture granuloma. Tracheal stenosis and/or malacia may develop 
at the closed tracheostomy site with consequent airway obstruction (35-37).

If the PTCF is small, $<4 \mathrm{~mm}$ in diameter, debridement and resection of the fistulous tract and primary closure may be effective. For larger defects (= or $>4 \mathrm{~mm}$ in diameter) the closure of PTCF with skin flap techniques is recommended. Direct closure or flap closure surgical techniques and outcomes may be variable in terms of morbidity and mortality. One of the recommended options to prevent flap surgery or a possible complication of direct closure has been described in a very simple way. In patients with large tracheocutaneous fistulas (a defect of the anterior tracheal wall of $>$ or $=4 \mathrm{~mm}$ diameter), excision of the fistula and placement of a smaller tracheotomy tube is recommended. After healing, a second attempt for decannulating and allowing for spontaneous closure could be planned. No complications were reported with this approach (36).

Simple closure techniques may be performed at the bedside and under local anesthesia, such as use of a turnover skin flap to cover the fistula (36). Since the flap remains connected to its original blood supply (as opposed to free flap), tissue oxygenation is well maintained.

Authors used a turnover skin flap to cover the fistula. All of the procedures were completed at bedside under local anesthesia. The fistulae were successfully closed and well healed without complications within 7 to 9 days (38).

Due to local scar hyperplasia and poor blood circulation, an ideal fistula closure is often difficult to achieve. Although several fistula-closing methods have been reported in the literature, it is the choice of performing surgeon's eventual decision. Some patients may need to undergo more complex and difficult surgical closure of the defects. By using a turnover flap plasty to repair delayed-healing fistula, good results may be achieved. Since the overturn flap is taken from fresh tissue, it maintains local blood supply very well. These procedures should be reserved for large fistulas, but mainly they should be the choice of operation for permanent trachea cutaneous fistula closure. They are described in the next part of this article.

\section{Closure of long-term tracheostomy}

The terms "long-term tracheostomy" or "permanent tracheostomy" do not necessarily mean 'irreversible'. When underlying disease is successfully treated, surgical closure of a permanent stoma may be an option. For example, a stoma may be used as an adjunct to major reconstruction for treatment of staged laryngotracheal reconstruction and may be surgically closed if the process has been successfully completed (39-41).

This part of this manuscript describes a long-term tracheostomy and its differences with PTCF. This two entities are different and they should be discussed in different ways although most of the articles in the literature presents combined and undifferentiated experiences. A tracheocutanous fistula should be evaluated as a pathologic complication of "temporary tracheostomy" resulting from failure of the tracheostomy tract to close spontaneously when the cannulae is intentionally withdrawn (25-34).

A tracheostomy may be referred to as "permanent", if it was intentionally performed in a way to shorten the distance between the trachea and the skin. This procedure involves removing all the tissues between trachea and skin, and thus establishes a tight circumferential permanent mucocutaneous junction (42-44). Long-term tracheostomy (LTT) offers prolonged maintenance of a tube-free, selfsustaining stoma. These stomas are preferred for neck cancer treatments. With the developments in surgery and oncology care, these patients survive and they require closure of their tracheostomies. Surgical techniques have been described in different ways. First of all, surgeons dealing with trachea should be aware of meticulous execution and proper planning are the landmarks for single-staged closure of a wide stoma with large crater like depression.

\section{General principles are as follows}

The technique should begin with resurfacing of the inside of the trachea with smooth epithelial-lined viable skin. This procedure should also provide the integrity of the tracheal walls at the level of the stoma. The flap should be viable, immediate and has continuous epithelial lining of the intraluminal defect in the anterior tracheal wall surface. By this way, it prevents future complications such as granulation tissue formation and tracheal stenosis.

It is recommended that the tracheal defect could be closed with a well-perfused, randomly vascularized skin flap at the site of the stoma. Meticulous hemostasis and mini-drains are used to prevent subcutaneous emphysema and hematoma formation around the stoma closure. Perioperative antibiotics prevent cellulitis, abscess formation, wound dehiscence, and septicemia.

Some advancement of viable, randomly vascularized muscle or subcutaneous fibroadipose tissue could be used over the skin flap to fill the craterlike depression at the 

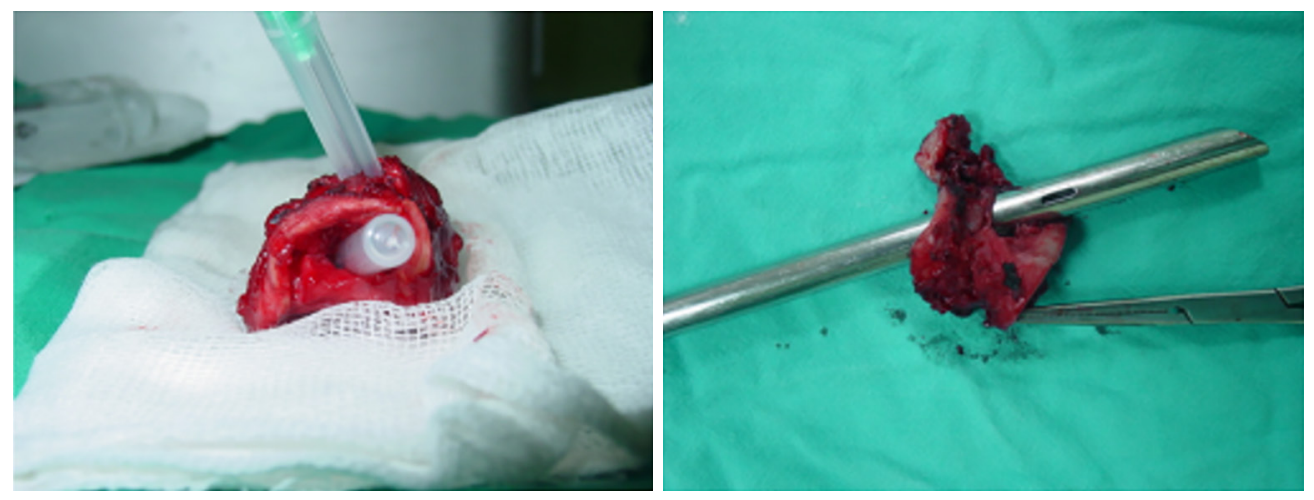

Figure 7 Dilatation of a stenosis is the first step of treatment in a patient. A tracheostomy or a reresection should not be performed if dilatation achieves success. Perforation of a trachea during dilatation may require an emergent operation.

stoma site. Primary tension-free closure of the skin results in consistently acceptable cosmetic results.

\section{Standard tracheal resection}

Postintubation tracheal stenosis (PITS) is the most common indication for standard tracheal resection. Excessively high tracheal cuff pressure, prolonged intubation and mobile tracheostomy tubes are causes of PITS. Injury to the tracheal mucosa causes ischemia, inflammation, fibrosis, and cicatrices contracture, resulting in tracheal stenosis. The use of high-volume, low-pressure tracheal cuffs has mitigated this complication, however, the problem still persists. Despite these developments and increasing awareness tracheal problems, secondary to mechanical ventilation and endotracheal intubation remains. The surgical techniques developed by Grillo are still the cornerstone of PITS treatment today (45).

\section{Etiology and diagnosis}

Dyspnea in a patient who has recently been extubated or decannulated should be considered to have PITS, until proven otherwise, and should undergo diagnostic evaluation. Symptoms may not be noticed for weeks or months. If they are severe or acute in onset, the patient should be evaluated immediately in the operating room for bronchoscopic evaluation and temporary treatment with dilatational bronchoscopy. Although it has been recommended to have flexible bronchoscopes, balloon dilators, rigid bronchoscopes, pediatric bronchoscopes, and instruments to perform an emergent tracheotomy, it is the authors of this manuscript preference to have rigid bronchoscope of size $3 \mathrm{~mm}$ OD to
$8 \mathrm{OD}$ and a ready tracheotomy set available (46). Authors prefer to start with pediatric size 5 rigid bronchoscopy and increase up to 8.5 gradually. In our experience an emergency tracheostomy never required but an emergency tracheal resection was performed for a transtracheal injury during dilatation (Figure 7).

\section{Operation planning and strategies}

Simply, dilatation is emergency but reconstructive surgery is almost never an emergency (47). Emergent bronchoscopy allows for direct visualization of tracheal anatomy. With further radiologic examinations, i.e., chest CT with three-dimensional reconstruction in the axial, coronal, and sagittal plans, the extent and location of tracheal injury may be mapped and assist with devising a treatment strategy (Figure 8). If it has not been performed before the operation, on the day of elective surgery, careful bronchoscopic dilation provides an airway for intubation with a size 6 ETT (47). Dilation should be done to allow intubation with a size of 6 endotracheal tube. It would be sufficient to start the procedure. During these bronchoscopies, endoscopists should be aware of airway inflammation, quality of the airway above and below the injury, presence of purulent secretions, and presence of additional or secondary stenosis within the trachea. Microbiology cultures taken before the surgery might be extremely valuable in the postoperative period, especially from patients who were hospitalized in other hospital/country ICUs. Measurement from the carinathe length of the stenosis - to the cricoid and the vocal cords define the lesion and the type of the operation to be performed. This mapping also helps to understand the risks of the procedure. The decision to proceed requires assessment of the status of the tracheal mucosa, the length of stenosis, 

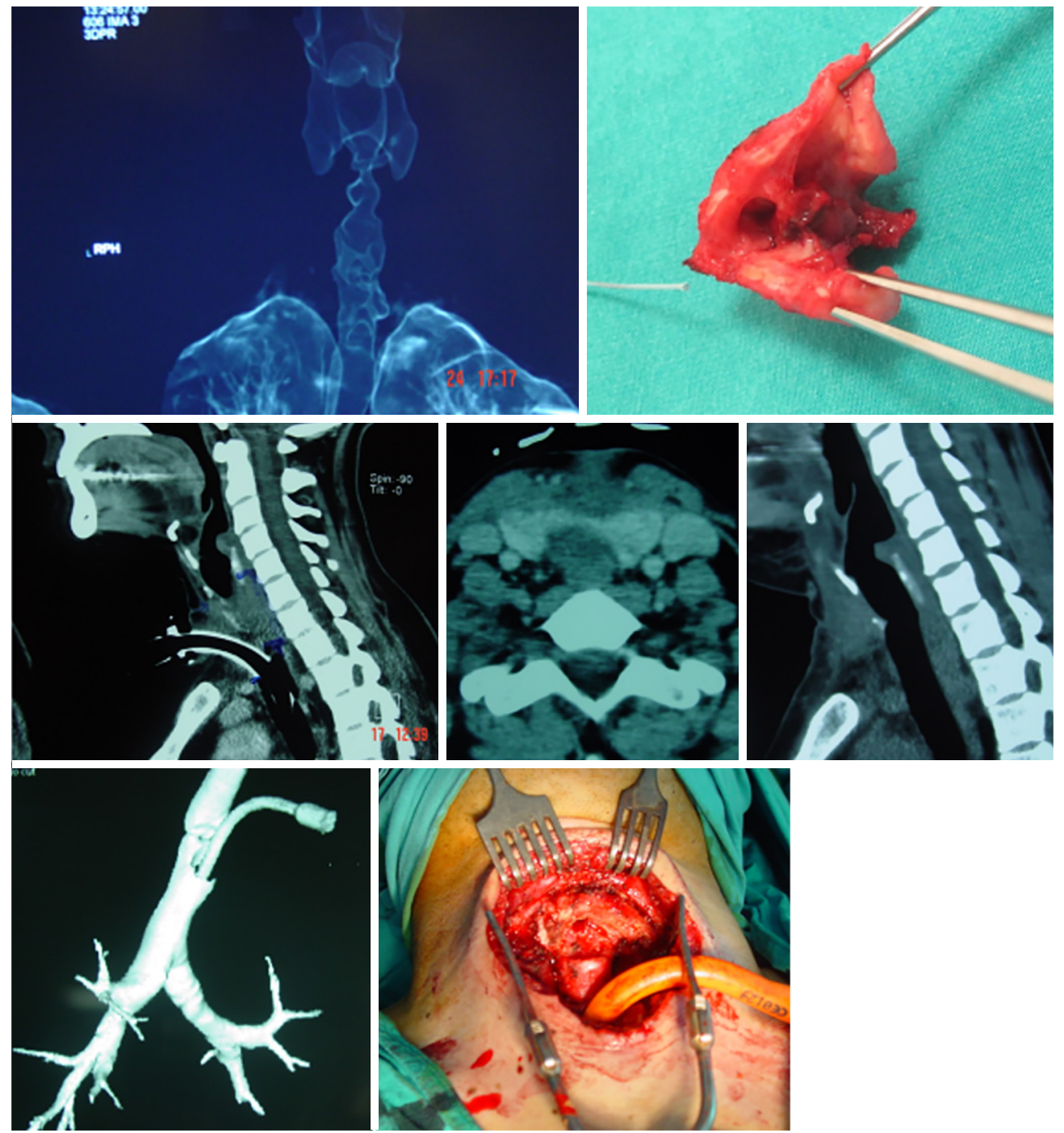

Figure 8 Chest CT with three-dimensional reconstruction in the axial, coronal, and sagittal plans, the extent and location of tracheal injury may be mapped and assist with devising a treatment strategy.

location, consideration of body habitus, prior treatments, and general overall condition of the patient (46). Four centimeters of stenosis can be resected safely, however, for greater length of resections, there may be a need to use release maneuvers such as the suprahyoid laryngeal release as the first option (45). Half of the adult trachea can be resected and reconstructed with the help of additional release maneuvers. It should be kept in mind that this refers to the ideal patients: healthy, long neck patient with addition of suprahyoid, pericardial release maneuvers in addition to the division of the carina and reimplantation of the major bronchi (45).

Neck extension is provided with a bag placed under the shoulders. The patient is prepared from chin to xiphoid process keeping in mind that a sternotomy may be required. In this operation anesthetists should coordinate with surgeons to ensure that patients are extubated before leaving the OR.

\section{Flow of operation}

Although the most common incision is a low collar incision, division of the manubrium may be required. A small sternum retractor may help in separating the manubrium and right side of the sternum could be broken to have wider 

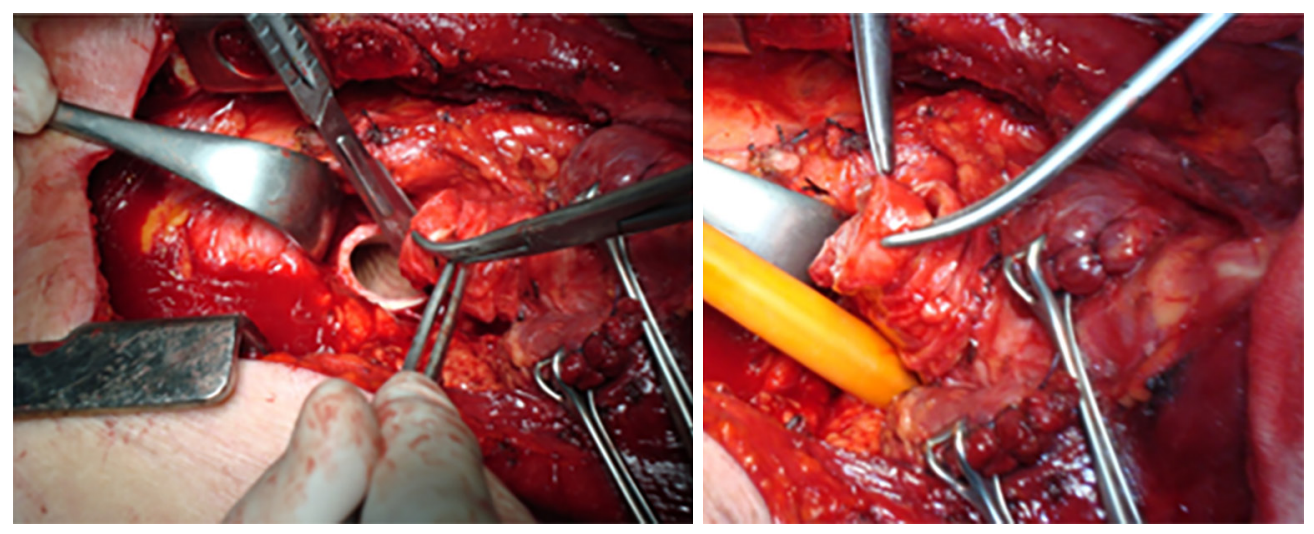

Figure 9 Every millimeter is important in tracheal resections.

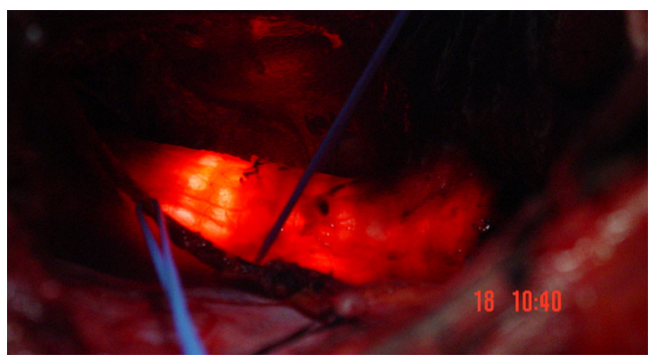

Figure 10 Operating room lights are turned off during bronchoscopy. So proper resection line could be planned. Remember "every mm is important".

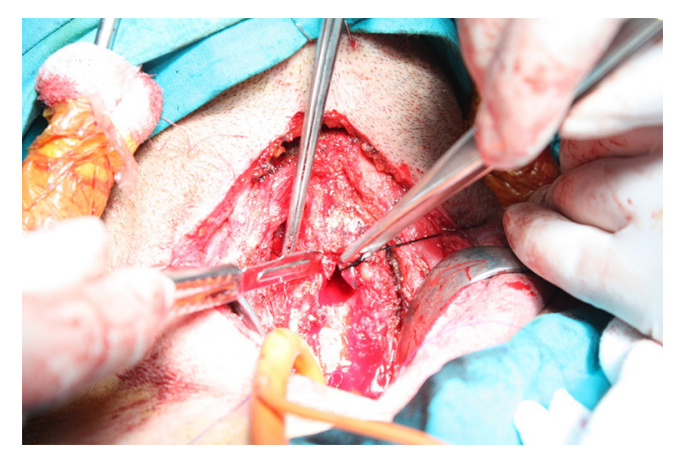

Figure 11 After the trachea is opened distally, a sterile ETT \#8 is connected to the ventilatory tubing and handed off to the anesthesiologist. Oral ETT tube is pulled back, but still keeping in the limits of upper tracheal resection, a silk tie is attached to the distal hole of this tube.

exposure. A full sternotomy is rarely required; however, it is very helpful for extended release maneuvers, such as bilateral pericardial release and separation of diaphragm from pericardium and major bronchial releases. It is standard start to develop superior skin flap to expose the thyroid cartilage. The thyroid isthmus is divided and suture ligated and retracted laterally to improve exposure. A pretracheal plane is dissected beyond the innominate artery as it has been done in mediastinoscopy. Surgeons should dissect only in the cartilaginous surface of the trachea without causing devascularisation to the cartilaginousmembranous part, where the feeding vessels enter into the trachea. Identification of location of stenosis and calculating the resection margins may require pediatric flexible bronchoscope.

The operating rooms light may be turned off so that the surgeon could identify the bronchoscopic light in the trachea and estimate the resection margins. Every millimeter is important in tracheal resections (Figures 9,10). The dissection of trachea in a lateral surface is kept on trachea, without entering in the paratracheal fatty tissue. This allows the recurrent nerves to fall away of the surgical site. Surgeons should not make any attempt to identify the nerves.

Cautery should be used with bipolar mode during the lateral dissection on the trachea wall.

Although some authors recommend to carry out the dissection circumferentially around the airway, it is the authors' preference to resect the membranous trachea and separate it from the esophagus without any attempt of circumferential dissection.

After the trachea is opened distally, a sterile ETT \#8 is connected to the ventilatory tubing and handed off to the anesthesiologist. Oral ETT tube is pulled back, but still keeping in the limits of upper tracheal resection, a silk tie is attached to the distal hole of this tube (Figure 11). That silk 


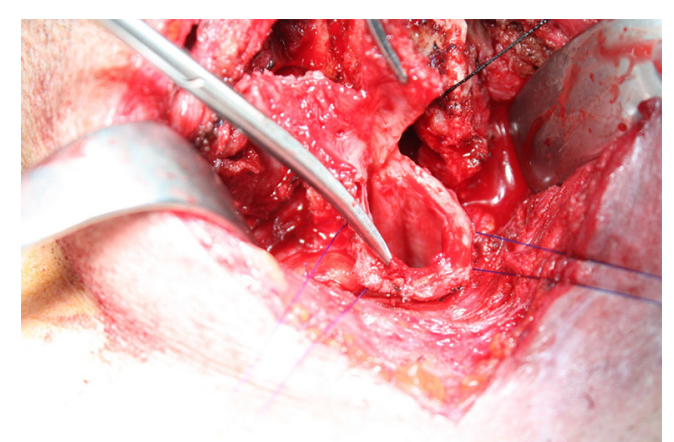

Figure 12 Traction sutures may be used both at proximal and distal sites.

allows easy retrieval of the ETT back in the trachea at the end of the procedure.

If the cuff of the tube is injured during the division of the trachea, use an aspiration cannula number 10 and place a strong silk suture, anesthesiologists pull it back and suture this silk to a new ETT \#8 Using this method, the intubation tube can be exchanged with a larger one.

The airway incision is performed through the most damaged part of the trachea. Resection of the damaged portion should be done by taking extreme care with each bite of trachea resection. Placement of a nasogastric tube may be helpful in identifying the esophagus. Resecting the trachea with precisely calculated margins is the most economic way to achieve anastomosis.

Traction sutures may be used both at proximal and distal sites (Figure 12). These sutures help to determine whether the trachea can be approximated with reasonable tension. If there exists a concern regarding excessive tension, release maneuvers should be considered. First choice is always a suprahyoid laryngeal release. A hyoid release should allow additional mobility of 1 to $1.5 \mathrm{~cm}$.

Recommended anastomotic sutures are 4-0 Vicryl, 4-0 PDS, or 4-0 Prolene. Currently the most commonly used suture is 4-0 PDS. We also have developed a technique with intermittent periods of apnea to perform a more secure and precisely approximated anastomosis. Our strategy is to stop ventilation and resume when pulse oximetry decreases to $92 \%$. The sutures are placed 3 to $4 \mathrm{~mm}$ deep with 3 to $4 \mathrm{~mm}$ between each suture. Place the first suture at the contralateral side of the surgeon at the deepest part and the posterior membranous part suture in a continuous way while the tube is removed. The rest of the sutures are single sutures. Each time a suture is placed, it is kept untied until the next suture is placed. Once the next suture is placed then the previous one is tied. This prevents unnecessary clamps and ties in the operating field.

Before the tying of last 3 to 4 sutures, the ETT cuff is deflated and the tube is pulled down towards the distal side of anastomosis (47). As the tube is placed distally, residual sutures are tied. The airway is carefully suctioned and, keeping the cuff deflated, the anesthesiologist is asked to close the mouth and obstruct the nasal orifices and perform a manual, high pressure ventilation.

The patient's neck is flexed until the chin is close to the manubrium. That suture should not be overtight. Spinal cord ischemia or other pathologies may develop due to excessive neck flexion.

Although suggested we generally do not perform control bronchoscopies if the patient does not have a symptom for any type of complication. The chin stitch is divided on postoperative day 5. Patients are cautioned to not extend their neck, lift excessive amounts of weight or drive for 3 to 4 weeks after surgery. Success is measured by adequacy of the airway and preservation of the voice.

\section{Acknowledgments}

Funding: None.

\section{Footnote}

Provenance and Peer review: This article was commissioned by the Guest Editor (Servet Bölükbas) for the series "Airway Surgery", published in fournal of Thoracic Disease. This article has undergone external peer review.

Conflicts of Interest: All authors have completed the ICMJE uniform disclosure form (available at http://dx.doi. org/10.21037/jtd.2020.02.41). The series "Airway Surgery" was commissioned by the editorial office without any funding or sponsorship. GA serves as the unpaid editorial board member of Fournal of Thoracic Disease from Apr 2019 to Mar 2021. The other authors have no other conflicts of interest to declare.

Ethical Statement: The authors are accountable for all aspects of the work in ensuring that questions related to the accuracy or integrity of any part of the work are appropriately investigated and resolved.

Open Access Statement: This is an Open Access article distributed in accordance with the Creative Commons 
Attribution-NonCommercial-NoDerivs 4.0 International License (CC BY-NC-ND 4.0), which permits the noncommercial replication and distribution of the article with the strict proviso that no changes or edits are made and the original work is properly cited (including links to both the formal publication through the relevant DOI and the license). See: https://creativecommons.org/licenses/by-nc-nd/4.0/.

\section{References}

1. Pierson DJ. Tracheostomy from A to Z: historical context and current challenges. Respir Care 2005;50:473-5.

2. McClelland RM. Tracheostomy: its management and alternatives. Proc R Soc Med 1972;65:401-4.

3. Jackson C. Tracheotomy. Laryngoscope 1909;19:285-90.

4. Durbin CG Jr. Early complications of tracheostomy. Respir Care 2005;50:511-5.

5. Epstein SK. Late complications of tracheostomy. Respir Care 2005;50:542-9.

6. Terragni PP, Antonelli M, Fumagalli R, et al. Early vs late tracheotomy for prevention of pneumonia in mechanically ventilated adult ICU patients: a randomized controlled trial. JAMA 2010;303:1483-9.

7. Young D, Harrison DA, Cuthbertson BH, et al. Effect of early vs late tracheostomy placement on survival in patients receiving mechanical ventilation: the TracMan randomized trial. JAMA 2013;309:2121-9.

8. Bittner EA, Schmidt UH. The ventilator liberation process: Update on technique, timing and termination of tracheostomy. Respir Care 2012;57:1626-34.

9. Delaney A, Bagshaw SM, Nalos M. Percutaneous dilatational tracheostomy versus surgical tracheostomy in critically ill patients: a systematic review and meta-analysis. Crit Care 2006;10:R55.

10. Higgins KM, Punthakee X. Meta-analysis comparison of open versus percutaneous tracheostomy. Laryngoscope 2007;117:447-54.

11. Dawood A, Haddad S, Arabi Y, et al. The safety of percutaneous tracheostomy in patients with coagulopathy or thrombocytopenia. Middle East J Anesthesiol 2007;19:37-49.

12. Auzinger G, O'Callaghan GP, Bernal W, et al. Percutaneous tracheostomy in patients with severe liver disease and a high incidence of refractory coagulopathy: a prospective trial. Crit Care 2007;11:R110.

13. Kluge S, Meyer A, Kühnelt P, et al. Percutaneous tracheostomy is safe in patients with severe thrombocytopenia. Chest 2004;126:547-51.
14. Byhahn C, Lischke V, Meininger D, et al. Perioperative complications during percutaneous tracheostomy in obese patients. Anaesthesia 2005;60:12-5.

15. Aldawood AS, Arabi YM, Haddad S. Safety of percutaneous tracheostomy in obese critically ill patients: a prospective cohort study. Anaesth Intensive Care 2008;36:69-73.

16. Romero CM, Cornejo RA, Ruiz MH, et al. Fiberoptic bronchoscopy-assisted percutaneous tracheostomy is safe in obese critically ill patients: a prospective and comparative study. J Crit Care 2009;24:494-500.

17. Heyrosa MG, Melniczek DM, Rovito P, et al. Percutaneous tracheostomy: a safe procedure in the morbidly obese. J Am Coll Surg 2006;202:618-22.

18. Blankenship DR, Kulbersh BD, Gourin CG, et al. Highrisk tracheostomy: exploring the limits of the percutaneous tracheostomy. Laryngoscope 2005;115:987-9.

19. Stamenkovic SA, Morgan IS, Pontefrac DR, et al. Is early tracheostomy safe in cardiac patients with a median sternotomy incision? Ann Thorac Surg 2000;69:1152-4.

20. Aggarwal S, Smailes S, Dziewulski P. Tracheostomy in burn patients revisited. Burns 2009;35:962-6.

21. Grillo HC. Surgery of the trachea and bronchi. 1st edition. Hamilton, Ontario: B.C. Deckers, 2004.

22. Carr SR, Friedberg JS. Tracheostomy. In: LoCicero J, Feins RH, Colson Y, et al. editors. General Thoracic Suurghery Shiels. 8th edition. Philadelphia: Wolters Kluwer, 2019:853-60.

23. Goldenberg D, Ari EG, Golz A, et al. Tracheotomy Complications: A Retrospective Study of 1130 Cases. Otolaryngol Head Neck Surg 2000;123:495-500.

24. Berenholz LP, Vail S, Berlet A. Management of tracheocutaneous fistula. Arch Otolaryngol Head Neck Surg 1992;118:869-71.

25. Drezner DA, Cantrell H. Surgical management of tracheocutaneous fistula. Ear Nose Throat J 1998;77:534-7.

26. Wheeler WB, Kurachek SC, Lobas JG, et al. Respiratory complications of tracheocutaneous fistula closure. Crit Care Med 1991;19:580-2.

27. Myers EN. Closure of tracheocutaneous fistula. In: Myers $\mathrm{EN}$, editor. Operative otolaryngology head and neck surgery. Philadelphia: Saunders, 2008.

28. Bressler KL, Kaiser PC, Dunham ME, et al. Primary closure of persistent tracheocutaneous fistula in children. Ann Otol Rhinol Laryngol 1994;103:835-7.

29. Licameli GR, Marsh BR, Tunkel DE. A simple method for closure of tracheocutaneous fistula in children. Arch 
Otolaryngol Head Neck Surg 1997;123:1066-8.

30. Goldsmith AJ, Abramson AL, Myssiorek D. Closure of tracheocutaneous fistula using a modified cutaneous Z-plasty. Am J Otolaryngol 1993;14:240-5.

31. Lewis VL, Manson PN, Stalnecker MC. Some ancillary procedures for correction of depressed adherent tracheostomy scars and associated tracheocutaneous fistula. J Trauma 1987;27:651-5.

32. Jacobs JR. Bipedicle delayed flap closure of persistent radiated tracheocutaneous fistulas. J Surg Oncol 1995;59:196-8.

33. Wheeler WB, Kurachek SC, Lobas JG, et al. Respiratory complications of tracheocutaneous fistula closure. Crit Care Med 1991;19:580-2.

34. Bressler KL, Kaiser PC, Dunham ME, et al. Primary closure of persistent tracheocutaneous fistula in children. Ann Otol Rhinol Laryngol 1994;103:835-7.

35. Mickelson SA, Rosenthal L. Closure of permanent tracheostomy in patients with sleep apnea: a comparison of two techniques. Otolaryngol Head Neck Surg 1997;116:36-40.

36. Drezner DA, Cantrell H. Surgical management of tracheocutaneous fistula. Ear Nose Throat J 1998;77:534-7.

37. Licameli GR, Marsh BR, Tunkel DE, A simple method for closure of tracheocutaneous fistula in children. Arch

Cite this article as: Toker A, Hayanga JA, Dhamija A, Herron R, Abbas G. Tracheotomy, closure of long-term tracheostomy and standard tracheal segmental resections. J Thorac Dis 2020;12(10):6185-6197. doi: 10.21037/jtd.2020.02.41
Otolaryngol Head Neck Surg 1997;123:1066-8.

38. Qilin Huang, Haipeng Liu, Shengqing Lü. A simple skin flap plasty to repair tracheocutaneous fistula after tracheotomy. Chinese Journal of Traumatology 2015;18:46-7.

39. Eliachar I, Zohar S, Golz A, et al. Permanent tracheostomy. Head Neck Surg 1984;7:99-103.

40. Eliachar I, Oringher SF. Performance and management of longterm tracheostomy. Op Tech Otolaryngol Head Neck Surg 1990;1:56-63.

41. Eliachar I. Unaided speech in long-term tube-free tracheostomy. Laryngoscope 2000;110:749-60.

42. Eliachar I, Zohar S, Golz A, et al. Permanent tracheostomy. Head Neck Surg 1984;7:99-103.

43. Eliachar I, Oringher SF. Performance and management of longterm tracheostomy. Op Tech Otolaryngol Head Neck Surg 1990;1:56-63.

44. Eliachar I. Unaided speech in long-term tube-free tracheostomy. Laryngoscope 2000;110:749-60.

45. Montgomery WW. Suprahyoid release for tracheal stenosis. Arch Otolaryngol 1974;99:255-60.

46. Mathisen DJ. How I teach it. Tracheal resection and reconstruction. Ann Thorac Surg 2017;103:1043-8.

47. Grillo HC. Surgical treatment of postintubation tracheal stenosis. J Thorac Cardiovasc Surg 1979;78:860-75. 